\title{
Portable real-time photoacoustic microscopy
}

Konstantin Maslov, Hao F Zhang, Lihong V. Wang

Konstantin Maslov, Hao F Zhang, Lihong V. Wang, "Portable real-time photoacoustic microscopy," Proc. SPIE 6437, Photons Plus Ultrasound: Imaging and Sensing 2007: The Eighth Conference on Biomedical Thermoacoustics, Optoacoustics, and Acousto-optics, 643727 (13 February 2007); doi: 10.1117/12.702227

SPIE. Event: SPIE BiOS, 2007, San Jose, California, United States 


\title{
Portable real-time photoacoustic microscopy
}

\author{
Konstantin Maslov, Hao F. Zhang, Lihong V. Wang* \\ Optical Imaging Laboratory, Department of Biomedical Engineering, Washington University in St. \\ Louis, MO 63123-1097
}

\begin{abstract}
To make a photoacoustic microscope (PAM) suitable for clinical applications, we have developed a portable PAM system with high frame rate. The probe for this portable PAM system is a small hand-held unit, which is connected with the rest of the system with an optical fiber and electrical cables. The probe can be maneuvered easily and pressed against the region of interest at various anatomical sites. By employing a tunable laser with a repetition rate up to $1 \mathrm{kHz}$, this portable PAM system has reduced its acquisition time to less than one second for each cross-sectional image. We have applied this system to animals as well as humans to acquire cross-sectional images with a maximum imaging depth of 5 $\mathrm{mm}$, a lateral resolution of better than $100 \mu \mathrm{m}$, and an axial resolution of $35 \mu \mathrm{m}$.
\end{abstract}

Keywords: medical and biological imaging, photoacoustic imaging, photoacoustic microscopy, functional imaging

\section{INTRODUCTION}

Photoacoustic (PA) imaging technique ${ }^{1-8}$ is a hybrid technology that obtains the internal distribution of optical energy deposition in biological tissues by detecting laser-induced ultrasonic waves, which are referred to as PA waves. Hence, it reveals physiologically specific optical absorption contrast. PA imaging takes advantage of the weaker ultrasonic scattering, whose magnitude is two orders of magnitude less than that of optical scattering ${ }^{9}$ to achieve high spatial resolution in deep biological tissues compared with traditional optical imaging ${ }^{10,11}$. In addition, PA imaging is able to extract certain functional parameters, such as the hemoglobin oxygenation saturation $\left(\mathrm{SO}_{2}\right)$, without using exogenous contrast agents by varying the optical wavelength of the excitation laser and conducting spectroscopic measurement.

In recent years, two major forms of PA imaging techniques have been developed: photoacoustic tomography (PAT) $)^{2,3,5}$ and photoacoustic microscopy ${ }^{4}$. In PAT, an unfocused ultrasound detector/array and an inverse reconstruction algorithm are usually employed to acquire cross-sectional or three-dimensional images of biological samples. In contrast,, PAM typically uses a spherically-focused ultrasonic detector, works in a reflection mode, and employs higher ultrasonic frequencies than PAT to achieve more flexibility in imaging objects with various spatial shapes (rather than circular objects) and higher spatial resolution.

A table-top dark-field illumination PAM ${ }^{12,13}$, which can achieve much improved image quality at greater depth compared with the previously published PAM, was developed recently in our lab. This PAM provides an axial resolution of $15 \mu \mathrm{m}$ and a lateral resolution of $45 \mu \mathrm{m}$ with a maximum imaging depth of $3 \mathrm{~mm}$ using an ultrasonic transducer whose frequency is centered at $50 \mathrm{MHz}$. Based on intrinsic optical absorbing pigments, such as hemoglobin and melanin, in biological tissues, the table-top PAM system has been applied to image the morphology of the subcutaneous microvasculature ${ }^{14}, \mathrm{SO}_{2}$ in the microvasculature ${ }^{13}$, skin melanoma tumors ${ }^{15}$, and acute skin burns ${ }^{16}$. When bioconjugated contrast agents are administered, PAM can also be applied to image molecular and genetic contrasts such as imaging of LacZ genetic expression ${ }^{17}$. Moreover, the table-top PAM has been demonstrated to be safe and can be applied to human imaging. However, low image acquisition speed and bulky stationary mechanical scanner make the table-top PAM system vulnerable to motion artifacts and limit its applications only to small animals and certain anatomical sites in humans. Moreover, it is difficult for PAM to study transient physiological processes at a low imaging speed.

Photons Plus Ultrasound: Imaging and Sensing 2007: The Eighth Conference on Biomedical Thermoacoustics, Optoacoustics, and Acousto-optics, edited by Alexander A. Oraevsky, Lihong V. Wang, Proc. of SPIE Vol. 6437, 643727, (2007) · 1605-7422/07/\$18 - doi: 10.1117/12.702227 
To overcome these limitations, we have developed a portable real-time PAM system with a hand-held probe. The lightweighted hand-held probe is connected to the laser system, the data-acquisition system, and the motor-controlling system through optical fiber and electrical cables. It can be pressed against region of interest at various anatomical sites in animals and humans and uses ultrasonic gel for acoustic coupling, which is operated in the same manners as does the traditional ultrasonography. This portable real-time PAM system extends the range of applicability of PAM to virtually all anatomical sites and to certain transient physiological processes. We have tested this portable PAM system by acquiring B-scan images from different locations in humans and have achieved comparable image quality to the tabletop PAM system.

\section{METHODOLOGY}

The portable hand-held PAM system is based on the proven design of the table-top PAM ${ }^{12}$ with several improvements. First, a new tunable dye laser (CBR-D, Sirah) pumped by an Nd:YLF laser (INNOSLAB, Edgewave), whose pulse duration is $5.8 \mathrm{~ns}$ and maximum pulse repetition rate can be up to $2 \mathrm{kHz}$, is employed as our excitation source. Previously, the low pulse repetition rate prevents PAM from imaging in high speed. For example, it takes $20 \mathrm{~s}$ to acquire a B-scan image that contains 200 A-lines at a repetition rate of $10 \mathrm{~Hz}$. In comparison, with this new laser system, the data acquisition time for the same B-scan image is reduced to less than 1 second, which corresponds to a B-scan frame rate of $\sim 1 \mathrm{~Hz}$. As a result, motion artifacts due to, for example, breathing or positioning instability of the hand-held probe are minimized. Moreover it is possible to image some dynamic physiological processes.

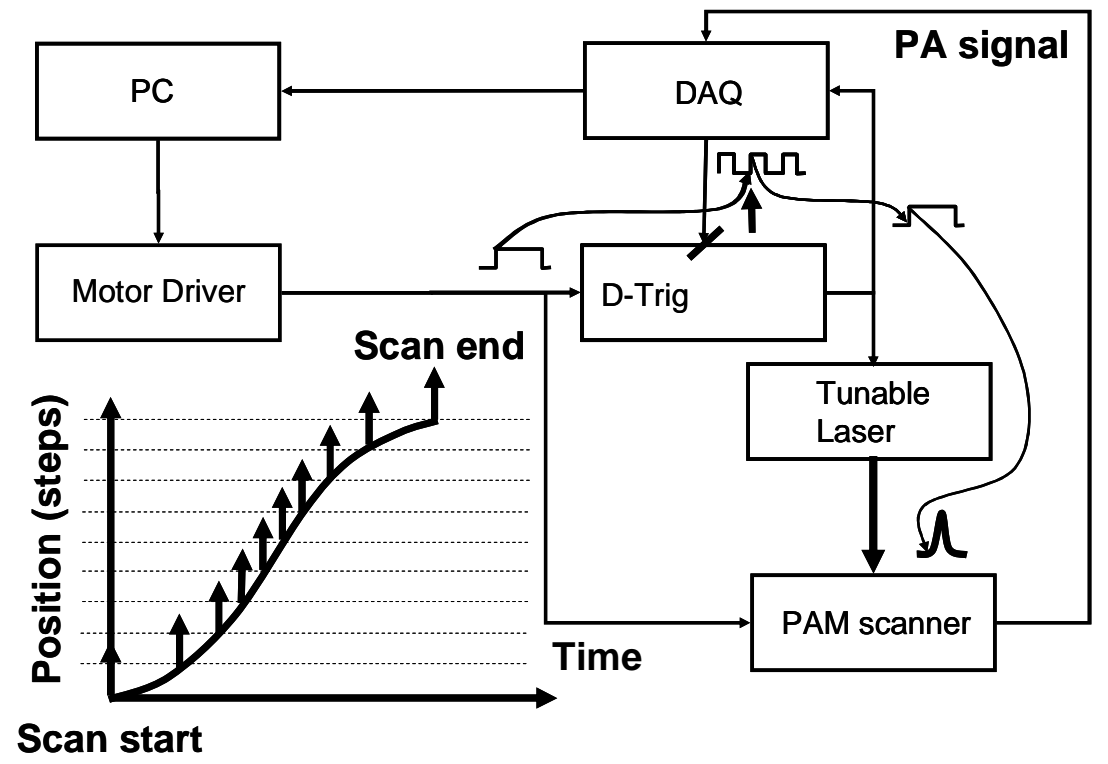

Fig. 1. Time diagram of the portable photoacoustic imaging device.

Second, we modify the data-acquisition system and the motor-controlling system (Fig. 1) in order to adapt for such a high pulse repetition rate. The data-acquisition system has been upgraded from a digital oscilloscope using a GPIB based data communication, whose data transfer speed is $0.6 \mathrm{MB} / \mathrm{s}$, to a digital acquisition (DAQ) board (CompuScope 12400, Gage Applied Technologies) using a PCI based data communication, whose data transfer speed is $132 \mathrm{MB} / \mathrm{s}$. The digital acquisition board has a dynamic range of $84 \mathrm{~dB}$ (14 bit) and a maximum data acquisition rate of 200MHz. Due to implementation of high pulse repetition rate laser and fast data acquisition system scanning speed of the portable scanner is limited by mechanical scanning system, correspondingly, compared with the previous setup where synchronization was controlled by the data-acquisition system, in the new setup it is done by motor-controlling PC. During B-scans, the motor works in a continuous mode and the motor-controlling PC generates synchronization pulses to both the Nd:YLF pump laser and the data-acquisition system to initiate the laser firing and the data acquisition according the current position of the ultrasonic transducer. The laser triggering is further synchronized with the clock output of the DAQ board to minimize the acquisition jitter. 
As demonstrated in Fig.1, the pulse repetition rate, which can be as high as $1 \mathrm{kHz}$, varies with the translation speed and the preset scanning step size. However, high laser pulse repetition rate rise concerns about laser safety. First, the delivered laser pulse energy is $0.6 \mathrm{~mJ}$ within the visible spectral range and the illumination area on the skin surface is 0.1 $\mathrm{cm}^{2}$, which generates an exposure of $6 \mathrm{~mJ} / \mathrm{cm}^{2}$. This laser exposure is within the safety limits $\left(20 \mathrm{~mJ} / \mathrm{cm}^{2}\right) \mathrm{defined} \mathrm{by} \mathrm{the}$ ANSI laser safety standards ${ }^{18}$. Second, based on the current scanning step size, illumination area, and the fact that no signal averaging is performed, the estimated maximum permissible pulse repetition rate is $13 \mathrm{kHz}$, ${ }^{19}$ which is much higher than the maximum pulse repetition rate used by the portable PAM system. As a result, the portable PAM system is safe for human applications.

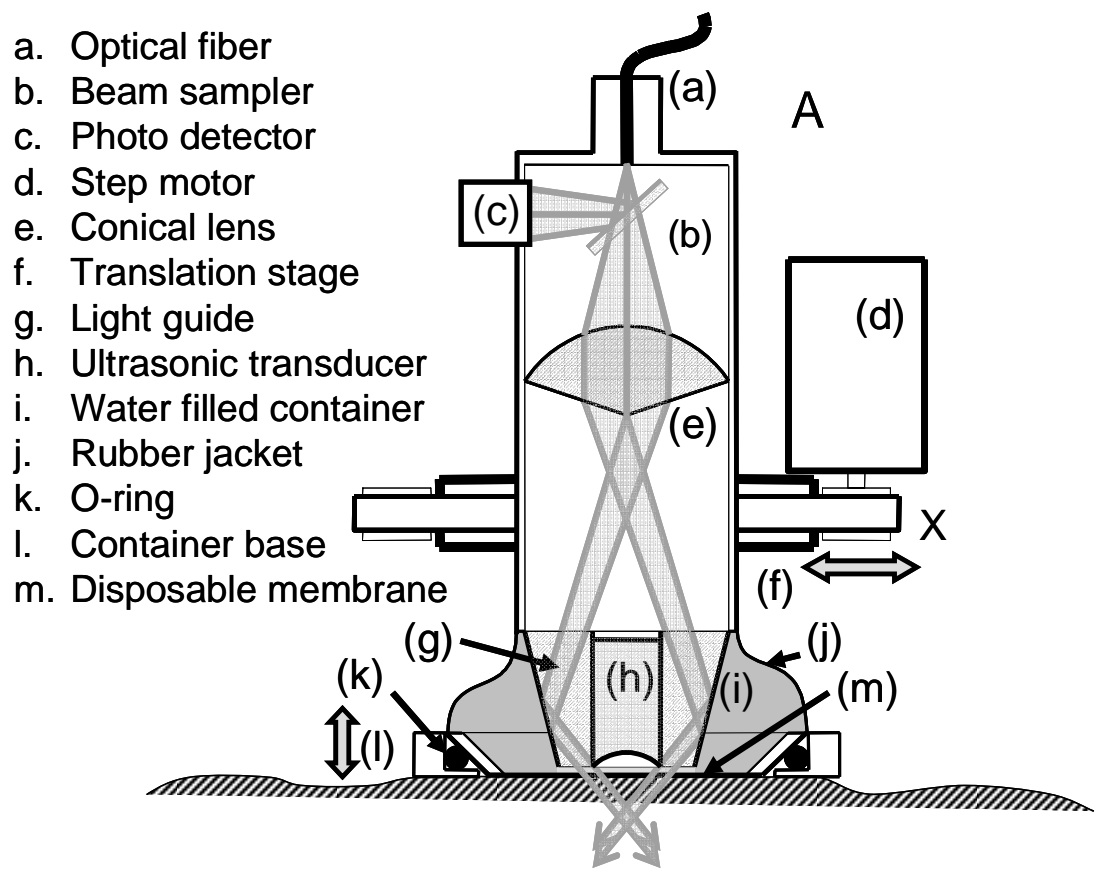

Fig. 2. Design of the hand-held photoacoustic scanner.

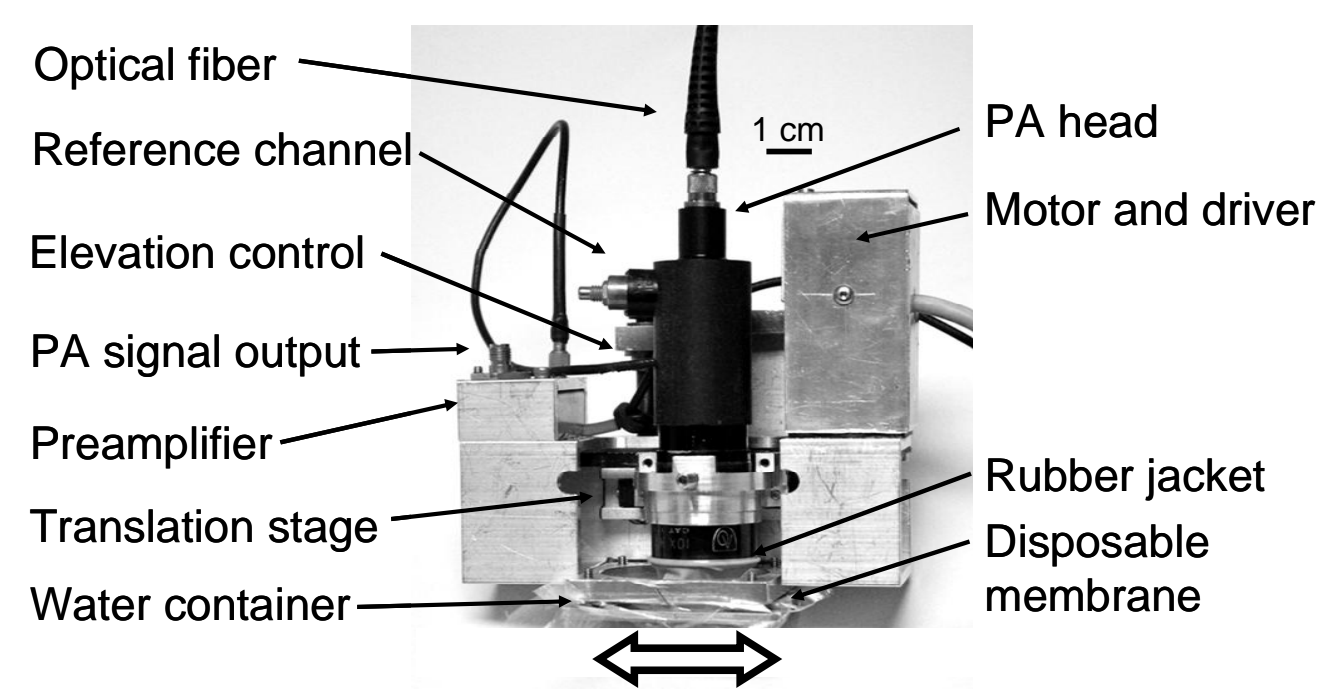

Fig. 3. Hand-held photoacoustic scanner. 
The design for the hand-held probe is schematically shown in Fig.2 and its photograph is shown in Fig.3. The probe consists of the following major components: an excitation optical fiber, a PA scan-head, a motorized translation stage, and a water-filled immersion chamber. The optical fiber delivers laser light to the hand-held scanner. The PA scan-head has the same basic design as the one used in the table-top PAM but is scaled down to fit in the miniature translation stage. The energy of each laser pulse is partially diverted to a photodiode to compensate for the pulse energy fluctuation. The transmitted laser beam passes through a conical lens to form a ring-shaped beam. It is then reflected by the outer wall of the conical light guide and weakly focused into the tissue with the focal region coaxially overlapping with the ultrasonic focus inside the tissue. The time-resolved PA signal is recorded at each location of the focused ultrasonic transducer for $5 \mu$ s and subsequently convert it into a 1D depth-resolved image (A-line).

The PA scan-head is mounted on a linear actuator, consisting of a belt driven translation stage and a NEMA-8 highresolution stepping motor. The ultrasonic transducer and the conical light guide are immersed in a water container. The scan-head can be vertically adjusted in the water container for ultrasonic focusing. A flexible rubber jacket is placed between the light guide and the container wall to seal the container. The bottom part of the container is sealed with an ultrasonically and optically transparent disposable polyethylene membrane, which is placed in direct contact with the object surface and pressed slightly toward the object surface. Acoustic coupling between the object surface and the membrane is provided by ultrasonic coupling gel. The disposable membrane is fixed in place by a quick release mechanism, which assures a waterproof seal and uniform tension across the membrane. The hand-held probe weights about 1lb, hence it can be moved around easily. Because it is watertight, it can be tilted to any orientation even upside down.

The ultrasonic transducer (V214-BB-RM, Panametrics, WA; element size: $6.35 \mathrm{~mm}$ ) has a 20-MHz center frequency and $80 \%$ nominal bandwidth. Focusing element is made of $5.2 \mathrm{~mm}$ radius of curvature $10 \mathrm{~mm}$ diameter plano-concave lens (CVI) attached to transducer waveguide. Assembled this way focusing ultrasonic transducer has numerical aperture (NA) of 0.43 , a focal length of $6.6 \mathrm{~mm}$, and a focal zone of $0.7 \mathrm{~mm}$. This PAM can provide an imaging depth of more than $5 \mathrm{~mm}$ in biological tissue, an axial resolution of $35 \mu \mathrm{m}$, and a lateral resolution of $100 \mu \mathrm{m}$ in its focal zone.

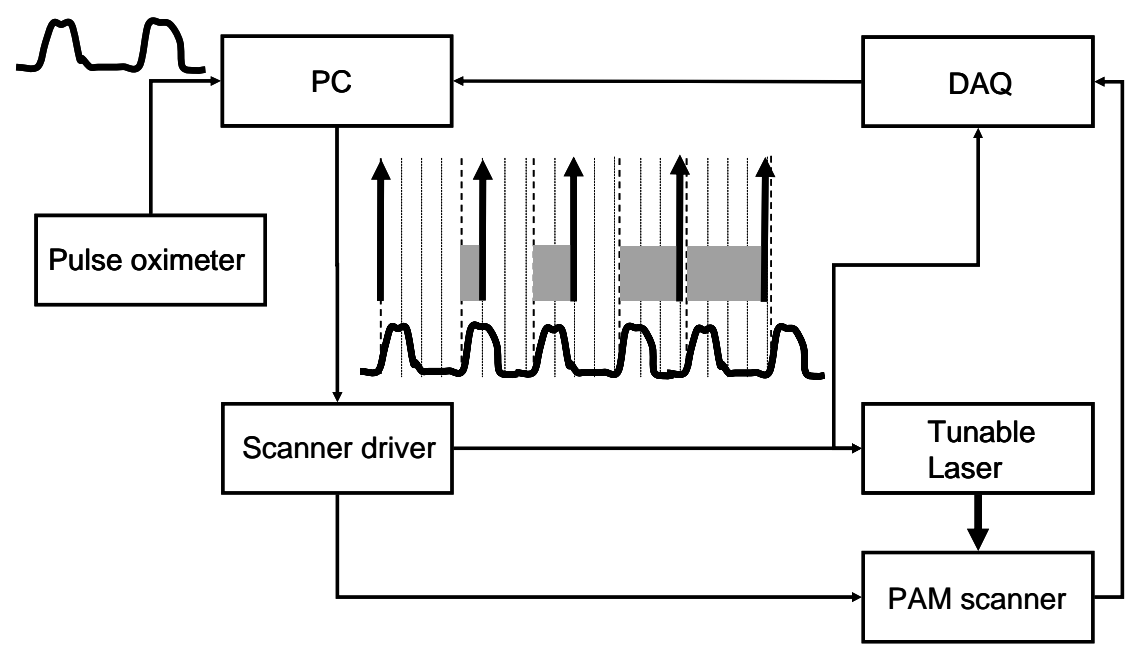

Fig.4. Pulse-wave synchronization (ECG triggering) of photoacoustic image.

For potential clinical applications, relatively fast scanning allows, for example, synchronization of image acquisition with cardiac cycle as shown in Fig. 4. This could diminish possible artifacts in quantitative imaging using PAM, such as the influence on PA amplitude caused by of blood vessel dilation in blood oxygen saturation measurements. In addition high imaging speed allows monitoring of cardiac cycle related processes. Even though $1-\mathrm{Hz}$ frame rate is not fast enough to acquire several images during single cardiac cycle, it is possible to obtain several images with starting point shifted in time with respect to, for example, P-wave peak of ECG or signal from heart rate monitor. In our experiment, we used the analogy output of a commercial pulse oximeter $(8600 \mathrm{~V}$, Nonin Medical, Inc) for this purpose. All experimental human procedures were carried out in conformity with the guidelines of the US National Institutes of Health. 


\section{RESULTS}

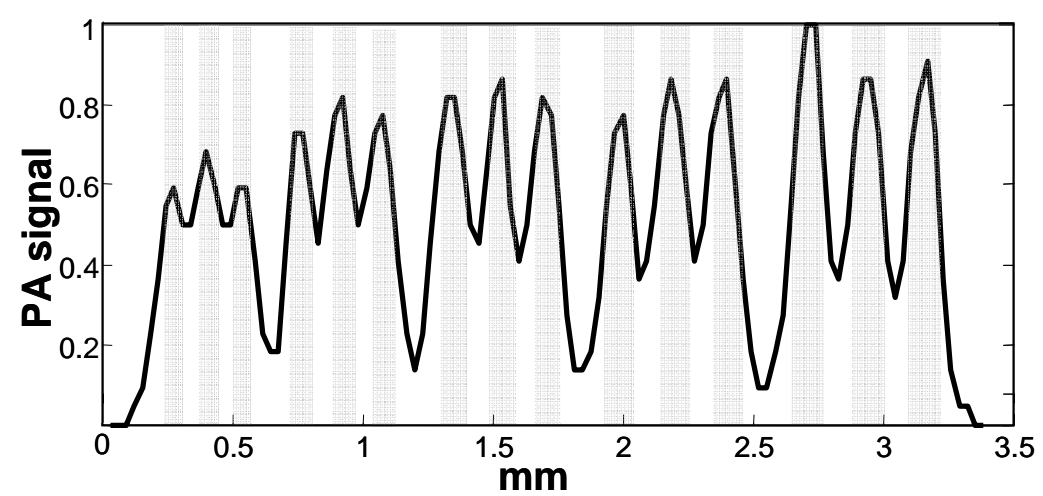

Fig.5. Transducer voltage as a function of transducer position with respect to USAF 1951 test target (shown as shadow strips).

We quantify the lateral resolution of the portable PAM system by imaging a Mylar USAF 1951 test target. In Fig. 5 PAM signal is plotted against the transducer position when it moves along test target (dark strips shown as shadowed areas). The modulation transfer function ${ }^{20}$, was extracted from the peak-to-peak amplitude of photoacoustic signal and extrapolated to the cut-off spatial frequency, producing an estimated lateral resolution of $100 \mu \mathrm{m}$. Such a lateral resolution was found to be comparable to that of the table-top PAM system provided a similar ultrasonic transducer. Axial resolution estimated from bandwidth of the photoacoustic signal is about $35 \mu \mathrm{m}$.
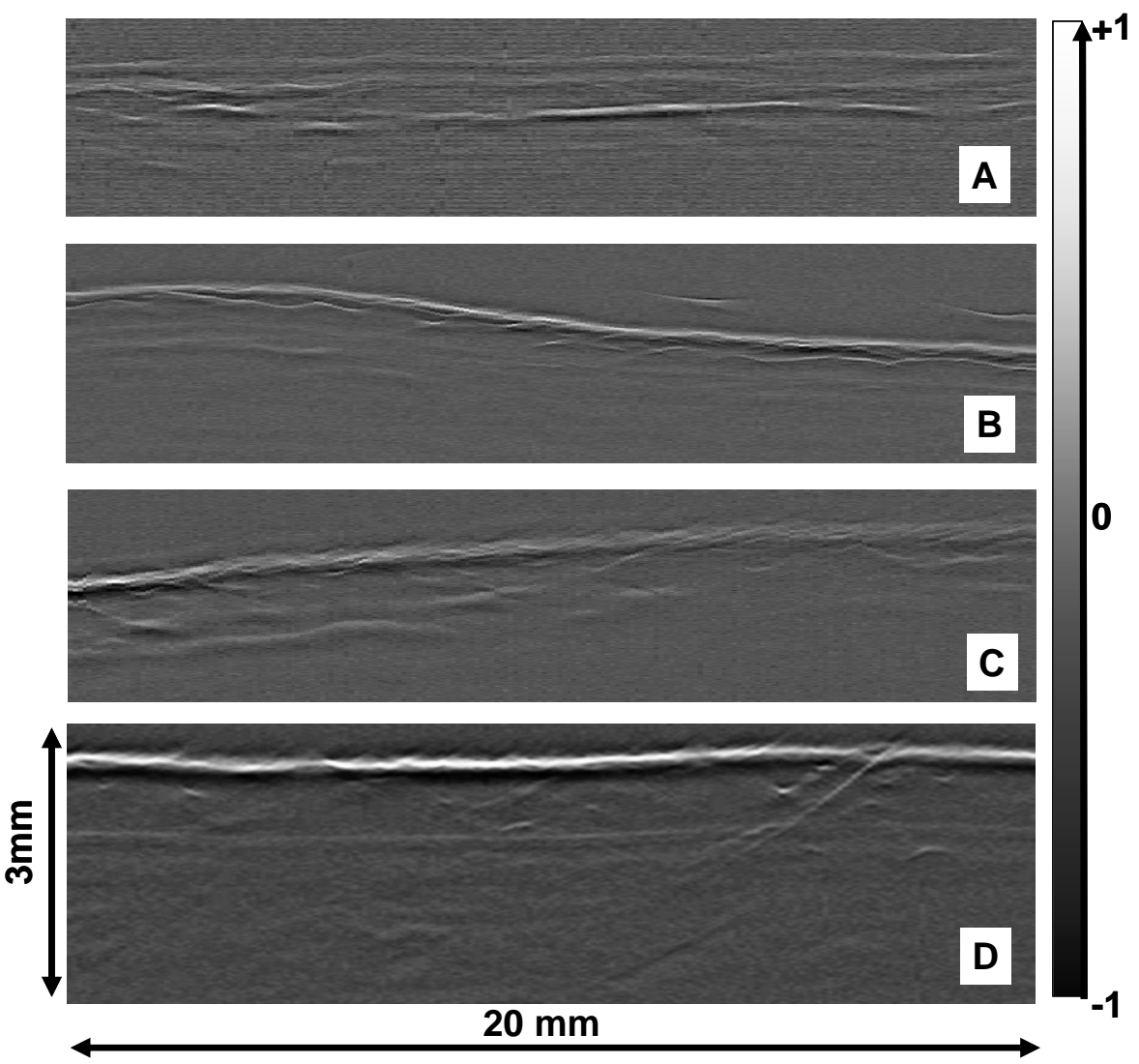

Fig.6. Cross-sectional PAM images of human skin. (A) palm dirsal area, (B) palm palmar area (C) foot planar area, (D) leg. 
Figure 6 shows B-scan images of the human skins in different locations. One can see significant difference in epidermis thickness and dermal vasculature in leg skin (Fig. 6C) compared with other images. Moreover, hair follicle surrounded by blood micro-vessels is shown in Fig. 6D. These images do not show observable distortion that was feared to be associated with the positioning instability of the hand-held probe. Hence, it proves the feasibility of building a clinically acceptable portable PAM system.
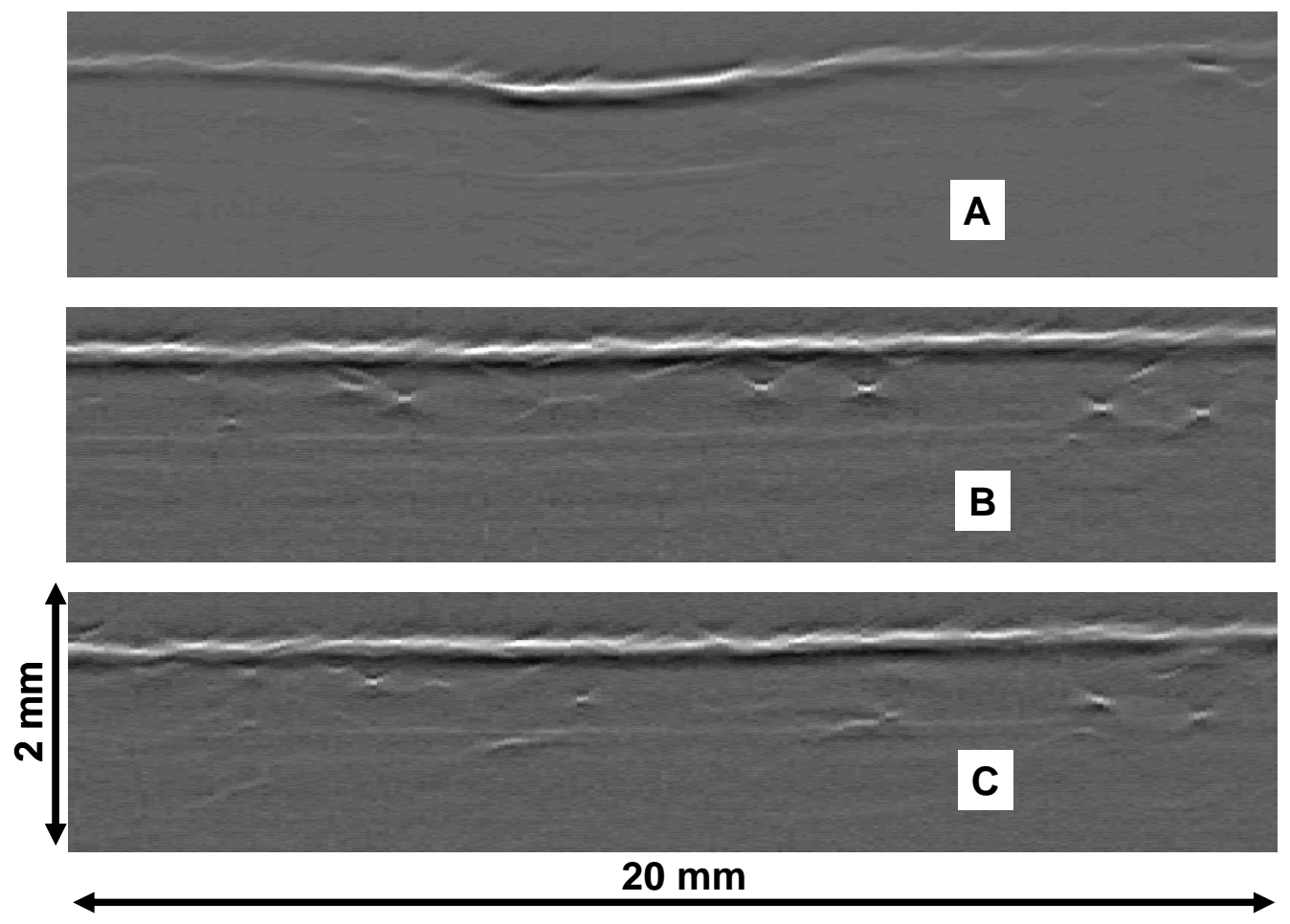

Fig.7. B-scan images of the microvasculature through the area of xerotic eczema (A) and a healthy skins location $1 \mathrm{~mm}$ apart (B) and (C).

Figure 7 shows B-scan PAM images of the microvasculature in a skin with a xerotic eczema and the adjacent health skins. One can find excessive pigmentation starting at epidermal layer and lack of blood vessel below in dermal and hypodermal layers in Fig. 7(A) compared with Fig. 7(B) and (C).

\section{DISCUSSION AND CONCLUSION}

In conclusion, we have developed a portable real-time PAM system equipped with a light-weighted hand-held probe and a high-repetition-rate tunable laser. The probe can be pressed against the area of interest and capable of acquiring one Bscan image in less than one second. Hand-held probe operates in the manner analogous to the traditional medical ultrasound probe, which facilitates the introduction of the imaging technology to the medical community. Based on the now laser system, the improved motor-controlling and data-acquisition systems, a frame rate of $1 \mathrm{~Hz}$ was achieved. Moreover this portable PAM has been tested on human skin in vivo and no motion artifacts or distortions were observed.

\section{ACKNOWLEDGEMENTS}

This project is sponsored by National Institutes of Health grants R01 EB000712 and R01 NS46214. 


\section{REFERENCES}

1. $\quad$ Xu, M. \& Wang, L. V. Photoacoustic imaging in biomedicine. Rev. Sci. Instrum. 77, 041101 (2006).

2. Wang, X. et. al. Noninvasive laser-induced photoacoustic tomography for structural and functional imaging of the brain in vivo. Nature Biotechnol. 21, 803-806 (2003).

3. Hoelen, C.G.A., de Mul, F.F.M., Pongers, R. \& Dekker, A. Three-dimensional photoacoustic imaging of blood vessels in tissue. Opt. Lett. 23, 648-650 (1998).

4. $\quad$ Oraevsky, A.A. \& Karabutov, A.A. Optoacoustic Tomography. in Biomedical Photonics Handbook, Vol. PM125 (ed. VoDinh, T.) CRC Press, Boca Raton, Florida, 2003.

5. Kruger, R.A., Liu, P., Fang, Y.R. \& Appledorn, C.R. Photoacoustic ultrasound (PAUS) - reconstruction tomography. Med. Phys. 22, 1605-1609 (1995).

6. Bell, A.G. On the production and reproduction of sound by light. Am. J. Sci. 20, 305-324 (1880).

7. Diebold, G.J., Khan, M.I. \& Park, S.M. Photoacoustic "signature" of particulate matter: optical production of acoustic monopole radiation. Science 250, 101-104 (1990).

8. Tam, A.C. Applications of photoacoustic sensing techniques, Rev. Mod. Phys. 58, 381-431 (1986).

9. Duck, F. A. Physical Properties of Tissue Academy Press London, 1990.

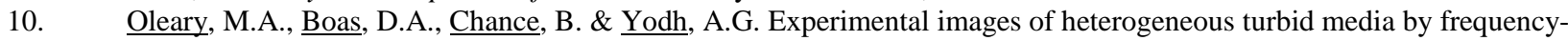
domain diffuse-photon tomography. Opt. Lett. 20, 426-428 (1995).

11. Boas, D.A., Brooks, D.H., Miller,_E.L., DiMarzio,_C.A., Kilmer,_M., Gaudette,_R.J. \&_Zhang, Q. Imaging the body with diffuse optical tomography. IEEE Signal Processing Magazine 18, 57-75 (2001).

12. Maslov, K., Stoica, G. \& Wang, L.V. In vivo dark-field reflection-mode photoacoustic microscopy. Opt. Lett. 30, 625-627 (2005).

13. Zhang, H.F., Maslov, K., Stoica, G \& Wang, L.V. Functional photoacoustic microscopy for high-resolution and noninvasive in vivo imaging. Nat. Biotechnol. 24, 848-851 (2006).

14. Zhang, H.F., Maslov, K., Li, M.-L., Stoica, G. \& Wang, L.V. In vivo volumetric imaging of subcutaneous microvasculature by photoacoustic microscopy. Opt. Express 14, 9317-9323 (2006).

15. Oh, J.-T., Li, M.-L., Zhang, H.F., Maslov, K., Stoica, G. \& Wang, L.V. Three-dimensional imaging of skin melanoma in vivo by dual-wavelength photoacoustic microscopy. J. Biomed. Opt. 11, 034032 (2006).

16. Zhang, H.F., Maslov, K., Stoica, G. \& Wang, L.V. Imaging acute thermal burns by photoacoustic microscopy. J. Biomed. Opt. 11, 054033 (2006).

17. Li, L., Zemp, R.J., Lungu, G., Stoica, G. \& Wang, L.V. Imaging of gene expression in vivo with photoacoustic tomography. Proceedings of the SPIE 6086, 62-67 (2006).

18. Laser Institute of America, American National Standard for Safe Use of Lasers ANSI Z136.1-2000, American National Standards Institute, Inc., New York, NY, 2000.

19. Zhang, H.F., Maslov, K. \& Wang, L.V. In vivo imaging of subcutaneous structures using functional photoacoustic microscopy. Nat. Protocols. Under revision (2007).

20. W. J. Smith, “Modern Optical Engineering," McGrew-Hill, New York, 1966, p. 318.

*lhwang@biomed.wustl.edu; phone 1314 935-6152; fax 1314 935-7448 